\title{
Stability analysis of output feedback control systems with memory-based event-triggering mechanism
}

\author{
Miguel A. Davó, Christophe Prieur, and Mirko Fiacchini
}

\begin{abstract}
This work is concerned with the stability analysis of an output feedback control system possibly influenced by unknown disturbances, where both the plant output and the controller output are subject to event-triggered sampling. We propose a new event-triggering mechanism based on the history of the measured outputs instead of the current outputs only. This novel feature provides a simple link between the parameters of the sampling criterion and the speed of convergence. Accumulation of sampling times is prevented by enforcing a minimum inter-event time. The effectiveness of the proposed event-triggered scheme is illustrated by several numerical examples, including nonlinear and linear systems.
\end{abstract}

\section{INTRODUCTION}

Sampled-data control for continuous-time dynamical systems is a very active research topic, in which a continuoustime plant is controlled with a digital device. Traditionally, the control task has been assumed to be executed periodically, which simplifies the implementation of the control system. However, the periodic sampling schemes may produce unnecessary updates of the sampled signals, which will cause high utilization of resources (e.g. computation time, communication bandwidth, etc.). To overcome that limitation, the eventtriggering approach was proposed, where the sampling actions are determined by some function of the system state, rather than by progression of time. Several experimental results, see [9], [19], [13], have shown the potential of the event-triggered control to reduce the number of samplings.

In the past few years, a multitude of strategies for eventtriggered control have been proposed, see [8], [15]. Some strategies are based on the difference between the current value of the state and the previous sample, see [14], [21], assuming in particular Input-to-State stability (ISS). Other more recent approaches require less strong assumptions and update the measure of the state only when a Lyapunov function has a sufficiently negative derivative, as the solution approaches to the equilibrium (see [18], [20]). Other techniques are based on an observer (or a norm-observer) and require the knowledge of the (sampled) output only, see [22] and [23]. Most of the work in the literature assumes that the full plant state is available, which is a strong assumption for many practical applications where only a part of the state can be directly measured. Generalizing event-triggered control techniques to output feedback control is definitively non-trivial, the simple

M. A. Davó, C. Prieur and M. Fiacchini are with Univ. Grenoble Alpes, CNRS, GIPSA-lab, F-38000 Grenoble, France. This work has been partially supported by the LabEx PERSYVAL-Lab (ANR-11-LABX-0025-01) and by the ANR project LimICoS contract number 12-BS03-005-01. Corresponding author: Miguel.Davo-Navarro@gipsa-lab.fr strategy [21] leads to Zeno phenomenon as shown in [3], [1]. Different event-triggering mechanisms have been proposed to solve this problem. For instance [12], [4] are based on state observers which lead to more complex event-triggering schemes. In [3], [17], the authors modified the triggering condition to guarantee an ultimate boundedness property instead of asymptotic stability. Another approach linked to time regularization technique is presented in [1], where timetriggered control and event-triggered control are combined to rule out Zeno phenomenon while asymptotic stability and ISS property are preserved.

In this note, we focus on the analysis of the internal stability and the input-to-output stability (IOS), under unknown disturbances, of nonlinear output feedback even-triggered control systems. In addition, we provide a procedure to upper bound the $\mathcal{L}_{\infty}$-gain of linear time-invariant (LTI) systems. We consider the scenario in which the sensor and the actuator are co-located, and both plant output and controller output are sampled synchronously. To provide asymptotic stability and IOS, we propose an event-triggering mechanism, where the sampling times are computed from the difference between the current plant output and controller output, and the last sample. A novel feature of the proposed mechanism is that the history of the outputs is used to determine the sampling times. Inspired by the results in [1], [16], the proposed triggering mechanism enforces a minimum inter-event time in order to avoid accumulation of the sampling times. Our stability analysis exploits techniques inspired by Lyapunov-Razumikhin theorem and Halanay's inequality (see e.g. [5]). For the particular case of LTI systems, the proposed exponential stability conditions and the procedure for computing the $\mathcal{L}_{\infty}$-gain upper bound are written in terms of Linear Matrix Inequalities (LMI). In addition, less conservative results in terms of the interevent times are developed by considering piecewise quadratic Lyapunov functions. The main advantage of our approach is the relation of the parameters of the sampling algorithm with the speed of convergence. Moreover, several examples suggest that these parameters are related with the inter-event times, leading to a tradeoff between the speed of convergence and the number of needed updates. A preliminary version of this work is [2], where a more restricted scenario is analyzed and no disturbances are considered.

The rest of the paper is organized as follows. First the problem under consideration and the event-triggered setup are introduced in Section II. Section III contains the stability analysis of nonlinear control systems. The results are particularized for LTI systems in Section IV. The proposed technique is illustrated by numerical examples in Section V. 
Notation: The sets $\mathbb{S}^{n}$ and $\mathbb{S}_{+}^{n}$ denote the sets of symmetric matrices of dimension $n \times n$ and the set of symmetric positive definite matrices of dimension $n \times n$, respectively. The notation $P>0$ for $P \in \mathbb{S}^{n}$ means that $P$ is positive definite $(P<0$ means negative definite). For a matrix $A \in \mathbb{R}^{n \times n}$, the notation $H e(A)$ refers to $A+A^{\top}$. For a symmetric matrix $A \in \mathbb{R}^{n \times n}$, $\lambda_{m}(A)$ and $\lambda_{M}(A)$ stand for the minimum and maximum eigenvalue, respectively. The notation $\|x\|$ is the Euclidean norm for $x \in \mathbb{R}^{n}$ and, for a function $f:[a, b] \rightarrow \mathbb{R}^{n}$, a norm is defined as $\|f\|:=\sup _{s \in[a, b]}\|f(s)\|$. A function $f: \mathbb{R}_{+} \rightarrow \mathbb{R}_{+}$is of class $\mathcal{K}$ if it is continuous, strictly increasing, and $f(0)=0$. The function $f$ is of class $\mathcal{K}_{\infty}$, if $f \in \mathcal{K}$ and $\lim _{s \rightarrow \infty} f(s)=\infty$. A continuous function $f: \mathbb{R}_{+} \times \mathbb{R}_{+} \rightarrow \mathbb{R}_{+}$is of class $\mathcal{K} \mathcal{L}$ if for each fixed $s$, the function $r \mapsto f(r, s)$ belongs to class $\mathcal{K}$ and for each fixed $r$, the function $s \mapsto f(r, s)$ is nonincreasing and $\lim _{s \rightarrow \infty} f(r, s)=0$. The space of essentially bounded measurable functions is denoted by $\mathcal{L}_{\infty}$.

\section{Problem Statement}

Consider an output-based control system formed by the feedback interconnection of a plant $\mathcal{P}$ and a controller $\mathcal{C}$. The plant is given by

$$
\mathcal{P}:\left\{\begin{array}{l}
\dot{x}_{p}(t)=f_{p}\left(x_{p}(t), u_{p}(t), w(t)\right) \\
y_{p}(t)=g_{p}\left(x_{p}(t)\right)
\end{array}\right.
$$

where $x_{p} \in \mathbb{R}^{n_{p}}$ is the state of the plant, $u_{p} \in \mathbb{R}^{n_{u p}}$ the control input applied to the plant, $w(t) \in \mathbb{R}^{n_{w}}$ an unknown disturbance and $y_{p} \in \mathbb{R}^{n_{y p}}$ the output of the plant. The controller is given by

$$
\mathcal{C}:\left\{\begin{array}{l}
\dot{x}_{c}(t)=f_{c}\left(x_{c}(t), u_{c}(t)\right) \\
y_{c}(t)=g_{c}\left(x_{c}(t)\right)
\end{array}\right.
$$

where $x_{c} \in \mathbb{R}^{n_{c}}$ is the state of the controller, $u_{c} \in \mathbb{R}^{n_{u c}}$ the input of the controller, and $y_{c} \in \mathbb{R}^{n_{y c}}$ the control signal. In addition, let assume that the feedback interconnection between the plant and the controller is affected by an exogenous signal $e(t):=\left[e_{y}^{\top}(t), e_{u}^{\top}(t)\right]^{\top} \in \mathbb{R}^{n_{e}}$ with $n_{e}:=n_{y p}+n_{y c}$, such that the interconnection is given by $u_{p}(t)=y_{c}(t)+e_{u}(t)$ and $u_{c}(t)=y_{p}(t)+e_{y}(t)$. Considering the state $x:=\left[x_{p}^{\top}, x_{c}^{\top}\right]^{\top} \in$ $\mathbb{R}^{n}$ with $n:=n_{p}+n_{c}$, the closed-loop system is described by

$$
\left\{\begin{array}{l}
\dot{x}(t)=f(x(t), e(t), w(t)), \\
z(t)=g(x(t))
\end{array}\right.
$$

where $z(t) \in \mathbb{R}^{n_{z}}$ is a performance variable and

$$
f(x, e, w):=\left[\begin{array}{l}
f_{p}\left(x_{p}, g_{c}\left(x_{c}\right)+e_{u}, w\right) \\
f_{c}\left(x_{c}, g_{p}\left(x_{p}\right)+e_{y}\right)
\end{array}\right] .
$$

The function $f$ is assumed to be continuous in all its arguments and $f(x, e, w)=0$ if $x=0, e=0, w=0$. The functions $g_{p}$ and $g_{c}$ are assumed be continuously differentiable, and there exists a function $\xi \in \mathcal{K}$ such that

$$
\left\|\left[g_{p}^{\top}\left(x_{p}\right), g_{c}^{\top}\left(x_{c}\right)\right]^{\top}\right\| \leq \xi(\|x\|) .
$$

The function $g$ is assumed to be continuous, and in addition, there exists a function $\xi_{z} \in \mathcal{K}$ such that $\|g(x)\| \leq \xi_{z}(\|x\|)$.
In order to derive the results in this work, the following assumption is considered:

Assumption 1: There exist a locally Lipschitz positive definite function $V: \mathbb{R}^{n} \rightarrow \mathbb{R}_{+}$, functions $\alpha, \bar{\alpha}, \alpha$, $\beta_{1 w} \in \mathcal{K}_{\infty}$, a locally Lipschitz positive semi-definite function $\beta_{e}: \mathbb{R}^{n_{e}} \rightarrow \mathbb{R}_{+}$, a real number $\theta>0$, a continuous function $H: \mathbb{R}^{n} \rightarrow \mathbb{R}_{+}$, and a continuous, nonnegative function $\delta: \mathbb{R}^{n_{y p}} \rightarrow \mathbb{R}_{+}$such that, for all $x \in \mathbb{R}^{n}$,

$$
\underline{\alpha}(\|x\|) \leq V(x) \leq \bar{\alpha}(\|x\|),
$$

and for all $e \in \mathbb{R}^{n_{e}}$, and $w \in \mathbb{R}^{n_{w}}$

$$
\begin{aligned}
\langle\nabla V(x), f(x, e, w)\rangle & \leq-\alpha(V(x))-H^{2}(x)-\delta\left(y_{p}\right) \\
& +\theta^{2} \beta_{e}^{2}(e)+\beta_{1 w}(\|w\|) .
\end{aligned}
$$

Remark 1: Assumption 1 is a $\mathcal{L}_{2}$-gain stability property ${ }^{1}$ of (3), which has been used for instance in [1], [16] with slight changes.

Consider the feedback interconnection of the plant (1) and the controller (2), where both the plant output and the controller output are made through a sampling mechanism. Therefore, the input of the plant and the controller are updated at some instants $t_{k}, k \in \mathbb{N}$, referred to as sampling times (or triggering times in the context of event-triggered control). In this way, the interconnection is given by

$$
u_{c}(t)=y_{p}\left(t_{k}\right), \quad u_{p}(t)=y_{c}\left(t_{k}\right)
$$

for all $t \in\left[t_{k}, t_{k+1}\right), k \in \mathbb{N}$.

The sampling times can be generated in several ways. In event-triggered control the sampling times are governed by event-triggered mechanisms, that continuously monitor the behavior of the plant and the controller, and generate events when some condition is satisfied. This work focuses on the emulation-based approach, where first the controller is designed to get some desired behavior for the continuous loop, and second, an event-triggering scheme is designed to provide a bounded deviation of the event-triggered implementation from the continuous one under Assumptions 1. Therefore, the problem is to design a sampling algorithm, i.e. the computation of the sequence $\left(t_{k}\right), k \in \mathbb{N}$, in order to guarantee stability properties of the system and at the same time to prevent Zeno solutions.

Let us define $\zeta(t):=\left[y_{p}^{\top}(t), y_{c}^{\top}(t)\right]^{\top}$, where $y_{p}(t)$ and $y_{c}(t)$ are the output of the plant and the controller of the system (3). The dynamics of the event-triggered closed-loop system can be described by (3) and (4), where now the exogenous signal $e: \mathbb{R}_{+} \rightarrow \mathbb{R}^{n_{e}}$ represents the samplinginduced error given by

$$
\begin{array}{ll}
e(t)=-\zeta(t) & t \in\left[0, t_{1}\right), \\
e(t)=\zeta\left(t_{k}\right)-\zeta(t) & t \in\left[t_{k}, t_{k+1}\right), k \in \mathbb{N}
\end{array}
$$

and whose evolution is governed between two consecutive sampling instants by $\dot{e}(t)=f_{e}(e(t), x(t), w(t))$ with

$$
f_{e}(e, x, w):=\left[\begin{array}{l}
-\frac{\partial}{\partial x_{p}} g_{p}\left(x_{p}\right) f_{p}\left(x_{p}, g_{c}\left(x_{c}\right)+e_{u}, w\right) \\
-\frac{\partial}{\partial x_{c}} g_{c}\left(x_{c}\right) f_{c}\left(x_{c}, g_{p}\left(x_{p}\right)+e_{y}\right)
\end{array}\right] .
$$

${ }^{1}$ Function $\beta_{1 w}$ in Assumption 1 can be defined as a continuous positive semi-definite function, but no improvement has been found for the purpose of this work. 
In order to develop the main results of this work, we extend the initial condition of the system (3) on the interval $[-h, 0]$ as follows: $x(t)=x(0), t \in[-h, 0]$, where $h>0$ will be a design parameter of the proposed event-triggered mechanism. The error signal is extended similarly, $e(t)=e(0), t \in[-h, 0]$. In addition, for the sake of simplicity, we define the function $V_{t}:[-h, 0] \rightarrow \mathbb{R}_{+}$given by $V_{t}(s)=V(x(t+s)), s \in[-h, 0]$, where $V(x(t))$ is the value of the Lyapunov function in Assumption 1 along the solution to the system for some initial condition $x(0)$ and disturbance $w$.

In order to force a minimum inter-event time in the line of [1], [16], an exponential growth condition on the samplinginduced error $e$ is assumed.

Assumption 2: There exist $\beta_{2 w} \in \mathcal{K}_{\infty}$ and real numbers $L_{1}, L_{2} \geq 0$ such that for all $x \in \mathbb{R}^{n}, e \in \mathbb{R}^{n_{e}}$, and $w \in \mathbb{R}^{n_{w}}$

$\left\langle\nabla \beta_{e}(e), f_{e}(e, x, w)\right\rangle \leq L_{1} \beta_{e}(e)+L_{2} H(x)+L_{2} \beta_{2 w}(\|w\|)$.

Remark 2: The technique proposed in this work is also applicable to a control system with a static output controller given by $y_{c}(t)=g_{c}\left(u_{c}(t)\right)$ where $u_{c} \in \mathbb{R}^{n_{u c}}$ and $y_{c} \in \mathbb{R}^{n_{y c}}$. In this case, for analysis purpose, it can be assumed that the controller is directly connected to the plant. Hence, the eventtriggered control system is modeled by (3) with $f(x, e, w):=$ $f_{p}\left(x_{p}, g_{c}\left(g_{p}\left(x_{p}\right)+e\right), w\right)$, where the error signal is given by (9) with $\zeta(t):=y_{p}(t)$ and its evolution between sampling instants is governed by $f_{e}(e, x, w):=-\frac{\partial}{\partial x_{p}} g_{p}\left(x_{p}\right) f_{p}\left(x_{p}, g_{c}\left(g_{p}\left(x_{p}\right)+\right.\right.$ $e), w)$.

\section{MAIN RESULTS}

In this section, we first present the proposed triggering mechanism. Second, asymptotic, exponential, and Input-toOutput stability criteria are provided for nonlinear systems.

\section{A. Memory-based event-triggered mechanism}

The proposed triggering condition is based on the results in [1], and the idea of memory-based event-triggering proposed in our recent work [2]. The sampling algorithm consists in checking when the sampling-induced error exceeds a bound involving a moving window of the history of the plant output and control signal. In addition, the algorithm prevents from Zeno phenomenon by imposing a minimum inter-event time. Consider a continuous positive definite function $\sigma: \mathbb{R}^{n_{e}} \rightarrow$ $\mathbb{R}_{+}$, which is assumed to satisfy

$$
\sigma(\zeta) \leq \beta_{V}(V(x))
$$

for all $x \in \mathbb{R}^{n}$ and some function $\beta_{V} \in \mathcal{K}$, then we propose the following sampling algorithm

$$
\begin{aligned}
& t_{k+1}:=\inf \left\{t>t_{k}+T\right. \text { such that } \\
& \left.\qquad \theta^{2} \beta_{e}^{2}(e(t)) \geq \delta\left(y_{p}(t)\right)+\max _{s \in[t-h, t]} \sigma(\zeta(s))\right\}
\end{aligned}
$$

with $0<T \leq \mathcal{T}(\eta, \theta, \lambda, L)$, where $\beta_{e}, \theta$ and $L=\left[L_{1}, L_{2}\right]$ are from Assumptions 1 and $2, \eta>0, \lambda \in(0,1), h>0$ and

$$
\mathcal{T}(\eta, \theta, \lambda, L):= \begin{cases}\frac{1}{L_{1} r_{1}} \arctan \left(r_{2}\right), & (1+\eta) \theta L_{2}>L_{1}, \\ \frac{1}{L_{1}} \frac{1-\lambda}{1+\lambda}, & (1+\eta) \theta L_{2}=L_{1}, \\ \frac{1}{L_{1} r_{1}} \operatorname{arctanh}\left(r_{2}\right), & (1+\eta) \theta L_{2}<L_{1}\end{cases}
$$

with

$$
r_{1}:=\sqrt{\left|\left(\frac{(1+\eta) \theta L_{2}}{L_{1}}\right)^{2}-1\right|}, \quad r_{2}:=\frac{r_{1}\left(1-\lambda^{2}\right)}{\frac{1}{L_{1}} \lambda(1+\eta) \theta\left(L_{2}^{2}+1\right)+1+\lambda^{2}} .
$$

The parameters $\eta, \lambda$, and $h$ are design parameters of the eventtriggering mechanism. The function $\mathcal{T}(\eta, \theta, \lambda, L)$ is based on a combination of the functions proposed in [1], [16]. The main difference is the constant $L_{2}$, which allows us to easily encompass the linear case by the nonlinear theory. In addition, note that the event-triggering algorithm proposed in [1] is directly obtained by setting $\sigma(\zeta)=0, L_{2}=1$ and $\lambda=0$. Moreover, if $L_{2}=1$, then as $\eta \rightarrow 0, \mathcal{T}(\eta, \theta, \lambda, L)$ approaches the maximum allowable transmission interval (MATI) given in [16]. In previous results based on ISS property, the sampling algorithm aims at keeping sufficiently small the samplinginduced error to guarantee that the Lyapunov function is strictly decreasing. However, the proposed algorithm aims at guaranteeing that the maximum of the Lyapunov function in a moving time window is decreasing. This allows local increments of the Lyapunov function while still ensuring the asymptotic convergence to zero.

\section{B. Stability analysis of nonlinear systems}

Definition 1: The trivial solution to the event-triggered control system (3) with (9), (13) and $w=0$ is

- stable if $\forall \varepsilon>0$, there exists a $\delta=\delta(\varepsilon)>0$ such that $\|x(0)\| \leq \delta$ implies $\|x(t)\| \leq \varepsilon$ for all $t \geq 0$;

- attractive if there exists a $\delta_{a}>0$ such that for any $\eta_{a}>0$ there exists $T:=T\left(\delta_{a}, \eta_{a}\right)$ such that $\|x(0)\| \leq \delta_{a}$ implies $\|x(t)\| \leq \eta_{a}$ for all $t \geq T$;

- asymptotically stable if it is stable and attractive;

- exponentially stable with decay rate $\gamma$ if there exist $\delta_{e}>0$ and $\eta_{e}>0$ such that $\|x(0)\| \leq \delta_{e}$ implies $\|x(t)\| \leq$ $\eta_{e} e^{-\gamma t}\|x(0)\|$ for all $t \geq 0$;

- globally asymptotically (respectively exponentially) stable if $\delta_{a}$ (respectively $\delta_{e}$ ) can be an arbitrarily large, finite number.

Definition 2: The event-triggered control system (3) with (9) and (13) is Input-to-Output stable if there exist functions $\beta \in \mathcal{K} \mathcal{L}$ and $\kappa \in \mathcal{K}$ such that

$$
\|z(t)\| \leq \max \left(\beta(\|x(0)\|, t), \kappa\left(\|w\|_{\infty}\right)\right)
$$

for all $t \geq 0$, where $z$ is the performance variable along the solution to the system with initial condition $x(0) \in \mathbb{R}^{n}$, and disturbance $w \in \mathcal{L}_{\infty}$.

Theorem 1: Under Assumptions 1 and 2, suppose there exist a continuous, nondecreasing function $\rho(s)>s$ and $a$ function $\varrho \in \mathcal{K}_{\infty}$ satisfying $\varrho\left(s_{1}+s_{2}\right) \leq \alpha\left(s_{1}\right)+\eta \theta \lambda s_{2}$ for all $s_{1}, s_{2} \geq 0$, such that the function $v$ defined by

$$
v: s \mapsto \varrho(s)-\beta_{V}(\rho(s))
$$

is of $\mathcal{K}$-class, then the event-triggered control system (3) with (9), (13), and $w=0$ is globally asymptotically stable. Moreover, if $\varrho$ and $\beta_{V}$ are Lipschitz continuous functions then system (3) is Input-to-Output stable. 
Proof: The first part of the proof is based on [1], [16], and some details are omitted. Consider a function $R(q)=V(x)+$ $\max \left(0, \theta \phi(\tau) \beta_{e}^{2}(e)\right)$ where $q=(x, e, \tau), \tau \in\left[t_{k}, t_{k+1}\right)$ for all $k \in \mathbb{N}$ is a clock variable introduced to describe the time elapsed since the last sampling instant, and $\phi$ is the solution to $\dot{\phi}=-2 L_{1} \phi-(1+\eta) \theta\left(L_{2}^{2} \phi^{2}+1\right)$ with $\phi(0)=\lambda^{-1}$. Consider the case $\phi(\tau) \geq 0$, then Assumptions 1 and 2, and the sampling algorithm (13) imply ${ }^{2}$

$$
\begin{aligned}
\dot{R}(q) & \leq-\alpha(V(x))-H^{2}(x)-\delta\left(y_{p}\right)+\theta^{2} \beta_{e}^{2}(e) \\
& +\beta_{1 w}(\|w\|)+\theta \beta_{e}^{2}(e)\left(-2 L_{1} \phi-(1+\eta) \theta\left(L_{2}^{2} \phi^{2}+1\right)\right) \\
& +2 \theta \phi \beta_{e}(e)\left(L_{1} \beta_{e}(e)+L_{2} H(x)+L_{2} \beta_{2 w}(\|w\|)\right) .
\end{aligned}
$$

Consider that $\delta\left(y_{p}\right) \geq 0$, then applying twice the fact that $2 a b \leq \frac{1}{\kappa} a^{2}+\kappa b^{2}$ for any $a, b \geq 0$, and $\kappa>0$, it follows

$$
\dot{R}(q(t)) \leq-\alpha(V(x(t)))-\eta \theta^{2} \beta_{e}^{2}(e(t))+\beta_{w}(\|w(t)\|),
$$

where $\beta_{w}(s)=\max \left(\beta_{w 1}(s), \frac{1}{\eta} \beta_{w 2}^{2}(s)\right)$. Considering the function $\varrho$ in (17), and the fact that $\phi(\tau) \leq \lambda^{-1}$ for all $\tau \geq 0$, it is obtained

$$
\dot{R}(q(t)) \leq-\varrho\left(R(q(t))+\beta_{w}(\|w(t)\|) .\right.
$$

Now let us consider the case $\phi(\tau) \leq 0$ then $\tau>T$ with $T$ from (13)-(15). First, we get $R(q)=V(x)$, then Assumption 1 and $H^{2}(x) \geq 0$ imply $\dot{R}(q(t)) \leq-\varrho(R(q(t)))-$ $\delta\left(y_{p}\right)+\theta^{2} \beta_{e}^{2}(e(t))+\beta_{w}(\|w(t)\|)$. Using the sampling mechanism (13), equation (12), the notation $V_{t}$, and the fact that $V(x) \leq R(q)$, it is obtained $\dot{R}(q(t)) \leq-\varrho(R(q(t)))+$ $\beta_{V}\left(\left\|R_{t}\right\|\right)+\beta_{w}(\|w(t)\|)$, where $R_{t}:[-h, 0] \rightarrow \mathbb{R}_{+}$is given by $R_{t}(s)=R(q(t-s)), s \in[-h, 0]$. Since $\beta_{V}\left(\left\|R_{t}\right\|\right) \geq 0$, the term $\beta_{V}\left(\left\|R_{t}\right\|\right)$ can be added to the term on the right of equation (20), and thus, we get

$$
\dot{R}(q(t)) \leq-\varrho\left(R(q(t))+\beta_{V}\left(\left\|R_{t}\right\|\right)+\beta_{w}(\|w(t)\|),\right.
$$

for all $t \geq 0$.

The rest of the proof is organized in two parts: first we prove the global asymptotic stability and second the Input-to-Output stability.

1) Proof of global asymptotic stability: Let consider $w=0$ and deal with stability and attractivity, separately.

- Stability: First, note that (5) implies that $\|(x(0), e(0))\| \leq \omega(\|x(0)\|)$ with $\omega \in \mathcal{K}$ given by $\omega(s):=\sqrt{s^{2}+\xi(s)^{2}}$. In addition, the fact that $\beta_{e}$ is continuous positive semi-definite and the inequality (6) imply that there exists a function $\bar{\alpha}_{R} \in \mathcal{K}_{\infty}$ such that $R(q) \leq \bar{\alpha}_{R}(\|(x, e)\|)$. Now, for any given $\varepsilon>0$, pick $\delta>0$ such that $0<\delta \leq \omega^{-1}\left(\bar{\alpha}_{R}^{-1}(\underline{\alpha}(\varepsilon))\right)$. Function $v$ satisfies the conditions in Proposition 3 (given in the appendix), and thus, Proposition 3 can be applied to function $R(q(t))$ with $\mu<R(q(0))$, which leads to $R(q(t)) \leq R(q(0)) \leq \bar{\alpha}_{R}(\omega(\delta)) \leq \underline{\alpha}(\varepsilon)$ for all $t \geq 0$. Therefore, it is obtained $\underline{\alpha}(\|x(t)\|) \leq V(x(t)) \leq R(q(t)) \leq \underline{\alpha}(\varepsilon)$ for all $t \geq 0$ and the proof of stability is complete.

\footnotetext{
${ }^{2}$ The notation $\dot{R}(q)$ should be understood as the generalized directional derivative of Clarke (see [1]).
}

- Attractivity: For any given $\delta_{a}, \eta_{a}>0$, let $\mu=\underline{\alpha}\left(\eta_{a}\right)$ and $\vartheta=\bar{\alpha}_{R}\left(\omega\left(\delta_{a}\right)\right)$, then $\|x(0)\| \leq \delta_{a}$ implies $R(q(0)) \leq \vartheta$. The application of Proposition 3 guarantees that there exists $T(\vartheta, \mu)$ such that $R(q(t)) \leq \mu$ for all $t \geq T(\vartheta, \mu)$. Therefore, it follows $V(x(t)) \leq R(q(t)) \leq \mu=\underline{\alpha}\left(\eta_{a}\right)$ for all $t \geq T\left(\bar{\alpha}_{R}\left(\omega\left(\delta_{a}\right)\right), \underline{\alpha}\left(\eta_{a}\right)\right)$, which completes the proof of attractivity.

The stability and the attractivity imply the asymptotic stability of the system. Since $\delta_{a}$ can be chosen arbitrarily large, the global asymptotic stability is proved.

2) Proof of Input-to-Output stability: Let define the function $\chi(s):=\varepsilon v(s)$, where $v$ is as in (17), for some $\varepsilon$ satisfying $0<\varepsilon<1$. For a given initial condition $x(0)$ and a disturbance $w$, let $\hat{t}:=\inf \left\{t>0: R(q(t)) \leq \chi^{-1}\left(\beta_{w}\left(\|w\|_{\infty}\right)\right)\right\}$ with $\hat{t}=\infty$ if $R(q(t))>\chi^{-1}\left(\beta_{w}\left(\|w\|_{\infty}\right)\right)$ for all $t>0$. Then (21) leads to $\dot{R}(q(t)) \leq-\varrho(R(q(t)))+\beta_{V}\left(\left\|R_{t}\right\|\right)+\chi(R(q(t)))$ for all $t \in[0, \hat{t})$. Lemma 2 with $\mu=\chi^{-1}\left(\beta_{w}\left(\|w\|_{\infty}\right)\right)$ (note that $\varrho(s)-\beta_{V}(\rho(s))-\chi(s)=(1-\varepsilon) v(s) \in \mathcal{K}$ ) guarantees that there exists a function $\beta \in \mathcal{K} \mathcal{L}$ such that $R(q(t)) \leq \max \left(\beta\left(R(q(0), t), \chi^{-1}\left(\beta_{w}\left(\|w\|_{\infty}\right)\right)\right)\right.$ for all $t \geq 0$. The bound of $R$ and the facts that $V(x) \leq R(q)$ and $\|z(t)\| \leq \xi_{z}(\|x(t)\|) \leq \xi_{z}\left(\underline{\alpha}^{-1}(V(x(t)))\right)$ lead to $\|z(t)\| \leq$ $\xi_{z}\left(\underline{\alpha}^{-1}\left(\max \left(\beta\left(\bar{\alpha}_{R}\left(\omega(\|x(0)\|, t), \kappa\left(\|w\|_{\infty}\right)\right)\right)\right)\right.\right.$ with $\kappa(s)=$ $\chi^{-1}\left(\beta_{w}(s)\right)$, and that completes the proof.

Theorem 2: Under Assumptions 1 and 2, for a given $\delta>0$, assume there exist positive scalars $\underline{k}, \bar{k}, k_{e}, k_{\xi}$ and $\lambda_{1}>\lambda_{2}$ such that

$$
\begin{array}{cc}
\underline{k} s^{2} \leq \underline{\alpha}(s), \quad \bar{k} s^{2} \geq \bar{\alpha}(s), \quad \beta_{e}(e) \leq k_{e}\|e\|, \\
\xi(s) \leq k_{\xi} s, \quad \lambda_{1} s \leq \alpha(s), \quad \lambda_{2} s \geq \beta_{V}(s)
\end{array}
$$

for all $0 \leq s \leq \delta$ and $e \in \mathbb{R}^{n_{e}}$ with $\|e\| \leq \delta$, where $\beta_{V}$ is as in (12), then the event-triggered control system (3) with (9), (13), $0<T \leq \mathcal{T}(\eta, \theta, \lambda, L)$ with $\eta \theta \lambda \geq \lambda_{1}$, and $w=0$ is locally exponentially stable with decay rate $\gamma>0$ given as the unique solution of

$$
2 \gamma=\lambda_{1}-\lambda_{2} e^{2 \gamma h} .
$$

In addition, if (22) holds for all $s \in[0, \infty)$ and all $e \in \mathbb{R}^{n_{e}}$, then the system is globally exponentially stable.

Proof: Let $\delta_{e}^{2}=\frac{2}{\bar{k}+k_{e}^{2} k_{\xi}^{2}} \min \left(\underline{k} \delta^{2}, \delta\right)$ and take $\varrho(s)=$ $\lambda_{1} s$. Considering inequality (21) with $w=0$ and the bounds (22), it follows $\dot{R}(q(t)) \leq-\lambda_{1} R(q(t))+\lambda_{2}\left\|R_{t}\right\|$ for all $t \geq 0$ and $\|x(0)\| \leq \delta_{e}$. Since $\lambda_{1}>\lambda_{2}$, then Lemma 1 (Halanay's inequality) can be applied to the above inequality. Hence, there exists $\gamma>0$ being the unique solution to (23) such that $R(q(t)) \leq R(q(0)) e^{-2 \gamma t}$. The bounds (22) lead to $\underline{k}\|x(t)\|^{2} \leq$ $V(x(t)) \leq R(q(t)) \leq\left(\bar{k}+\theta \lambda^{-1} k_{e}^{2} k_{\xi}^{2}\right)\|x(0)\|^{2} e^{-2 \gamma t}$. Therefore, the system (3) with (9) and (13) is exponentially stable with decay rate $\gamma$.

Remark 3: Theorem 2 provides a relation between the parameters of the sampling algorithm and the decay rate. First, note that from (23), the decay rate decreases when the parameter $h$ increases. In addition, $0.5\left(\lambda_{1}-\lambda_{2}\right)$ is the supremum of $\gamma$, that is $\lim _{h \rightarrow 0} \gamma(h)=0.5\left(\lambda_{1}-\lambda_{2}\right)$, where $\gamma(h)$ is the solution of (23) as a function of $h$. In addition, function $\sigma$ is related with $\gamma$ through the function $\beta_{V}$ and $\lambda_{2}$. 
On the other hand, small values of $\eta$ and $\lambda$ may be desirable to increase the minimum of the inter-event times, however this leads to small values of $\lambda_{1}$, and thus, small values of the decay rate through equation (23).

\section{APPLICATION TO LTI SYSTEMS}

In this section, we focus on a closed-loop system formed by an LTI plant given by

$$
\mathcal{P}:\left\{\begin{array}{l}
\dot{x}_{p}(t)=A_{p} x_{p}(t)+B_{p} u_{p}(t)+B_{p w} w(t), \\
y_{p}(t)=C_{p} x_{p}(t)
\end{array}\right.
$$

and an LTI controller described as follows:

$$
\mathcal{C}:\left\{\begin{array}{l}
\dot{x}_{c}(t)=A_{c} x_{c}(t)+B_{c} u_{c}(t), \\
y_{c}(t)=C_{c} x_{c}(t),
\end{array}\right.
$$

where $A_{p}, B_{p}, C_{p}, B_{p w}, A_{c}, B_{c}$, and $C_{c}$ are matrices of appropriate dimensions. The dynamics of the event-triggered closed-loop system can be described as

$$
\left\{\begin{array}{l}
\dot{x}(t)=(A+B C) x(t)+B e(t)+B_{w} w(t), \\
z(t)=C_{z} x(t),
\end{array}\right.
$$

where $C_{z}$ is some nonzero matrix of appropriate dimensions that defines the performance variable, $e(t)$ is given by (9), and

$$
\begin{array}{rlr}
A:=\left[\begin{array}{cc}
A_{p} & 0 \\
0 & A_{c}
\end{array}\right], & B:=\left[\begin{array}{cc}
0 & B_{p} \\
B_{c} & 0
\end{array}\right], \\
C:=\left[\begin{array}{cc}
C_{p} & 0 \\
0 & C_{c}
\end{array}\right], & B_{w}:=\left[\begin{array}{c}
B_{p w} \\
0
\end{array}\right] .
\end{array}
$$

For the analysis of the above system, the general Assumptions 1 and 2 are replaced by an asymptotic stability assumption on the LTI system.

Assumption 3: The controller $\mathcal{C}$ renders the system (26) with $e(t)=0$ and $w(t)=0$ for all $t \geq 0$ asymptotically stable, and thus, for every matrix $Q \in \mathbb{S}_{+}^{n}$ there exists a matrix $P \in \mathbb{S}_{+}^{n}$ such that

$$
-Q=(A+B C)^{\top} P+P(A+B C) .
$$

Definition 3: The $\mathcal{L}_{\infty}$-gain of the event-triggered control system (26) with (9) and (13) is defined as

$\kappa:=\inf \left\{\hat{\kappa} \in \mathbb{R}_{+}: \exists \varphi \in \mathcal{K}\right.$ s.t. $\|z\|_{\infty} \leq \hat{\kappa}\|w\|_{\infty}+\varphi(\|x(0)\|)$

$$
\left.\forall x(0) \in \mathbb{R}^{n}, \forall w \in \mathcal{L}_{\infty}\right\} \text {, }
$$

where $z$ is the performance variable of the solution to (26) with initial condition $x(0) \in \mathbb{R}^{n}$, and disturbance $w \in \mathcal{L}_{\infty}$.

Henceforth, the functions $\sigma$ and $\beta_{e}$ for the sampling algorithm (13) will be given by $\sigma(\zeta):=\sigma_{c} \zeta^{\top} U_{\sigma} \zeta$ and $\beta_{e}^{2}(e):=$ $e^{\top} U_{e} e$ with $\sigma_{c}>0$ and $U_{\sigma}, U_{e} \in \mathbb{S}_{+}^{n_{e}}$.

Proposition 1: For given scalars $\gamma, h, \sigma_{c}, \eta>0, \lambda \in$ $(0,1)$, with $\eta \lambda \geq 2 \gamma+\sigma_{c} e^{2 \gamma h}$ and matrices $U_{\sigma}, U_{e} \in \mathbb{S}_{+}^{n_{e}}$, assume there exist matrices $P \in \mathbb{S}_{+}^{n}$, and $U_{w} \in \mathbb{S}_{+}^{n_{w}}$, and real numbers $\varsigma_{1}, \varsigma_{2}>0$ such that

$$
\begin{gathered}
C^{\top} U_{\sigma} C-P \leq 0, \\
\left(2 \gamma+\sigma_{c}\left(e^{2 \gamma h}-1\right)\right) P-\varsigma_{1} C_{z}^{\top} C_{z} \geq 0, \\
\Psi+\operatorname{diag}\left(\left(2 \gamma+\sigma_{c} e^{2 \gamma h}\right) P+\varsigma_{2} \Pi, 0,0\right)<0
\end{gathered}
$$

hold, where $\Pi=(A+B C)^{\top} C^{\top} C(A+B C), Q$ is given by (28), and

$$
\Psi:=\left[\begin{array}{ccc}
-Q & P B & P B_{w} \\
\star & -U_{e} & 0 \\
\star & \star & -U_{w}
\end{array}\right]
$$

then for all positive real value $0<T \leq \mathcal{T}(\eta, 1, \lambda, L)$ and

$$
L_{1}=\frac{\sqrt{\left\|U_{e}\right\|}\|C B\|}{\sqrt{\lambda_{m}\left(U_{e}\right)}}, L_{2}=\max \left(\sqrt{\frac{\left\|U_{e}\right\|}{\varsigma_{2}}}, \frac{\sqrt{\left\|U_{e}\right\|}\left\|C B_{w}\right\|}{\sqrt{\eta\left\|U_{w}\right\|}}\right),
$$

the event-triggered control system given by (26), (9), and (13) with $w=0$ is globally exponentially stable with decay rate $\gamma$. Moreover, the $\mathcal{L}_{\infty}$-gain of the system is smaller than or equal to $\sqrt{\frac{1}{\varsigma_{1}}\left\|U_{w}\right\|}$.

Proof: Consider the Lyapunov function $V(x)=x^{\top} P x$, with time-derivative along the solutions to (26) given by $\dot{V}(x(t))=-x^{\top}(t) Q x(t)+H e\left(x^{\top}(t) P\left(B e(t)+B_{w} w(t)\right)\right)$. Adding $e^{\top}(t) U_{e} e(t)-e^{\top}(t) U_{e} e(t)$ and $w^{\top}(t) U_{w} w(t)-$ $w^{\top}(t) U_{w} w(t)$ with $U_{e} \in \mathbb{S}_{+}^{n_{e}}, U_{w} \in \mathbb{S}_{+}^{n_{w}}$, it is obtained $\dot{V}(x(t)) \leq \varphi^{\top} \Psi \varphi+e^{\top} U_{e} e+\left\|U_{w}\right\|\|w\|^{2}$, where $\varphi:=$ $\left[x^{\top} e^{\top} w^{\top}\right]^{\top}$. In addition, condition (32) implies

$$
\dot{V}(x(t)) \leq-\lambda_{1} V(x)-\varsigma_{2} x^{\top} x+e^{\top} U_{e} e+\left\|U_{w}\right\|\|w(t)\|^{2}
$$

with $\lambda_{1}=\left(2 \gamma+\sigma_{c} e^{2 \gamma h}\right)$. Note that condition (30) leads to $\sigma(\zeta) \leq \beta_{V}(V(x))$ with $\beta_{V}(s)=\sigma_{c} s$. Moreover, Assumptions 1 and 2 are satisfied by considering the following functions and real numbers: $\alpha(s)=\lambda_{1} s, H(x)=\sqrt{\varsigma_{2}}\|C(A+B C) x\|$, $\theta=1, \beta_{1 w}(s)=\left\|U_{w}\right\| s^{2}, \beta_{2 w}^{2}(s)=\eta\left\|U_{w}\right\| s^{2}, \delta\left(y_{p}\right)=0$. Therefore, the exponential stability with decay rate $\gamma>0$ is concluded by applying Theorem 2 .

In order to obtain an upper bound of the $\mathcal{L}_{\infty}$-gain, let us consider equation (21) from the proof of Theorem 1, with the above functions and real numbers:

$$
\dot{R}(q(t)) \leq-\left(2 \gamma+\sigma_{c} e^{2 \gamma h}\right) R(q(t))+\sigma_{c}\left\|R_{t}\right\|+\left\|U_{w}\right\|\|w(t)\|^{2} .
$$

Consider some $\varepsilon$ such that $e^{2 \gamma h}>\varepsilon>1$, then equation (21) leads to $\dot{R}(q(t)) \leq-\varepsilon \sigma_{c} R(q(t))+\sigma_{c}\left\|R_{t}\right\|$, whenever $R(q(t)) \geq \frac{\left\|U_{w}\right\|}{2 \gamma+\sigma_{c}\left(e^{2 \gamma h}-\varepsilon\right)}\|w\|_{\infty}^{2}$. Applying Proposition 3 with $v_{1}(s)=\varepsilon \sigma_{c} s, v_{2}(s)=\sigma_{c} s$, and $\rho(s)=\frac{1+\varepsilon}{2} s$, it is obtained

$$
R(q(t)) \leq \max \left(\frac{\left\|U_{w}\right\|}{2 \gamma+\sigma_{c}\left(e^{2 \gamma h}-\varepsilon\right)}\|w\|_{\infty}^{2}, R(q(0))\right) .
$$

Choosing $\varepsilon>1$ sufficiently close to 1 , it follows that (31) implies $\left(2 \gamma+\sigma_{c}\left(e^{2 \gamma h}-\varepsilon\right)\right) P-{ }_{\varsigma} C_{z}^{\top} C_{z} \geq 0$, and in addition, $\|z(t)\|^{2} \leq \frac{1}{\varsigma_{1}}\left(2 \gamma+\sigma_{c}\left(e^{2 \gamma h}-\varepsilon\right)\right) R(q(t))$ for all $t \geq 0$, which yields to the upper bound of the $\mathcal{L}_{\infty}$-gain, $\kappa \leq \sqrt{\frac{1}{\varsigma_{1}}\left\|U_{w}\right\|}$, since $2 \gamma+\sigma_{c}\left(e^{2 \gamma h}-1\right)>2 \gamma+\sigma_{c}\left(e^{2 \gamma h}-\varepsilon\right)$.

Remark 4: Note that for any system (26) satisfying Assumption 3, we can always find $\gamma, \sigma_{c}, h, U_{\sigma}$ and $U_{e}$, such the LMIs (30), (31), and (32) are feasible. For instance, let us set $U_{\sigma}=\frac{\lambda_{m}(P)}{\left\|C^{\top} C\right\|}$ then the LMI (30) is feasible. In addition, LMI (31) is feasible for $\varsigma_{1} \leq\left(2 \gamma+\sigma_{c}\left(e^{2 \gamma h}-1\right) \frac{\lambda_{m}(P)}{\left\|C_{z}^{\top} C_{z}\right\|}\right.$. Finally, by taking $U_{e}=\alpha_{e} I$ and $U_{w}=\alpha_{w} I$ with $\alpha_{e}, \alpha_{w}>0$, and applying the Schur complement twice on (32), it follows that 
the LMI (32) is feasible if

$$
\begin{gathered}
Q-\left(2 \gamma+\sigma_{c} e^{2 \gamma h}\right) P-\varsigma_{2} \Pi-\frac{1}{\alpha_{e}} P B B^{\top} P>0, \\
Q-\left(2 \gamma+\sigma_{c} e^{2 \gamma h}\right) P-\varsigma_{2} \Pi-\frac{1}{\alpha_{e}} P B B^{\top} P-\frac{1}{\alpha_{w}} P B_{w} B_{w}^{\top} P>0,
\end{gathered}
$$

with $\Pi=H e(C(A+B C))$, which hold for sufficiently large $\alpha_{e}$ and $\alpha_{w}$, and sufficiently small $\gamma, \sigma_{c}$, and $\varsigma_{2}$.

The conditions in Proposition 1 are obtained by using a quadratic Lyapunov function, which leads to a conservative stability criterion, specially for unstable open-loop systems. A simple relaxation of the quadratic Lyapunov functions consists in dividing the state space in different regions and considering a quadratic Lyapunov function for each region, leading to a piecewise quadratic Lyapunov function (see [10]). In order to divide the state space, we consider a uniform partition of the $\mathbb{R}^{2}$ subspace which leads to a partition of the $\mathbb{R}^{n}$ space through an orthogonal projection defined by a matrix $\Upsilon \in$ $\mathbb{R}^{2 \times n}$ (the results may depend on the election of $\Upsilon$ ). For the sake of the simplicity, the following result only deals with the exponential stability, although the estimation of the $\mathcal{L}_{\infty}$-gain can be tackled with the same approach.

Proposition 2: For given scalars $\gamma, h, \sigma_{c}, \eta>0, \lambda \in$ $(0,1)$, with $\eta \lambda \geq 2 \gamma+\sigma_{c} e^{2 \gamma h}$, matrices $U_{\sigma}, U_{e} \in \mathbb{S}_{+}^{n_{e}}$, and $\Upsilon \in \mathbb{R}^{2 \times n}$, assume there exist matrices $P_{i} \in \mathbb{S}_{+}^{n}$, scalar $\varsigma>0$, and scalars $\varrho_{1_{i}}, \varrho_{2_{i}} \geq 0, i=1, \ldots, N$ such that

$$
\begin{array}{cc}
C^{\top} U_{\sigma} C-P_{i}+\varrho_{1_{i}} S_{i}<0, & \\
{\left[\begin{array}{cc}
-Q_{i}+\left(2 \gamma+\sigma_{c} e^{2 \gamma h}\right) P_{i}+\varsigma \Pi+\varrho_{2_{i}} S_{i} & P_{i} B \\
\star & -U_{e}
\end{array}\right]<0}
\end{array}
$$

for $i=1, \ldots, N, \Gamma_{1}^{\top}\left(P_{N}-P_{1}\right) \Gamma_{1}=0$, and

$$
\Gamma_{i}^{\top}\left(P_{i}-P_{i-1}\right) \Gamma_{i}=0, \quad i=2, \ldots, N
$$

hold, where $\Pi=(A+B C)^{\top} C^{\top} C(A+B C), Q_{i}$ is given by (28) for each $P_{i}, \Gamma_{i}$ is a basis of the null space of $\Theta_{i} \Upsilon$ with $\Theta_{i}:=\left[\sin \left(\frac{i \pi}{N}\right)-\cos \left(\frac{i \pi}{N}\right)\right]$ and $S_{i}:=$ $-\Upsilon^{\top}\left(\Theta_{i}^{\top} \Theta_{i-1}+\Theta_{i-1}^{\top} \Theta_{i}\right) \Upsilon$, then for all positive real value $0<T \leq \mathcal{T}(\eta, 1, \lambda, L)$ with $L_{1}=\frac{\sqrt{\left\|U_{e}\right\|}\|C B\|}{\sqrt{\lambda_{m}\left(U_{e}\right)}}, L_{2}=\sqrt{\frac{\left\|U_{e}\right\|}{\varsigma}}$, the event-triggered control system given by (26), (9), and (13) with $w=0$ is globally exponentially stable with decay rate $\gamma$.

Proof: The proof follows as the proof of Proposition 1 by considering the Lyapunov function

$$
V(x)=x^{\top} P_{i} x, \quad \text { if } x^{\top} S_{i} x \geq 0, \quad i=1, \ldots, N,
$$

and by adapting [10].

\section{EXAMPLES}

\section{A. Nonlinear example}

In this example, we consider the controlled Lorenz equations (see [24]) given by the functions $f_{p}\left(x_{p}\right)=$ $\left[-a x_{1}+a x_{2}, b x_{1}-x_{2}-x_{1} x_{3}+u_{p}, x_{1} x_{2}-c x_{3}\right]^{\top^{\top}} \quad$ and $g_{p}(x)=x_{1}$ with $a, b, c>0$ and $x_{p}=\left[x_{1}, x_{2}, x_{3}\right]^{\top}$. The plant is controlled by a static output feedback controller given by $g_{c}\left(u_{c}\right)=-\left(\frac{p_{1}}{p_{2}} a+b\right) u_{c}$ (see Remark 2), where $p_{1}, p_{2}>0$. Consider the ISS Lyapunov function $V(x)=p_{1} x_{1}^{2}+p_{2} x_{2}^{2}+p_{2} x_{3}^{2}$. By taking suitable values of

\begin{tabular}{lccc}
\hline$\sigma_{c} h$ & 0.5 & 1 & 2 \\
\hline 0.5 & $0.0095(0.26,2.69)$ & $0.0187(0.24,2.68)$ & $0.0287(0.19,1.77)$ \\
1 & $0.0112(0.17,2.68)$ & $0.0193(0.15,2.66)$ & $0.0315(0.11,1.64)$ \\
2 & $0.0151(0.03,2.68)$ & $0.0213(0.02,2.53)$ & $0.0317(0.01,1.47)$ \\
\hline
\end{tabular}

TABLE I

EFFECT OF $\sigma_{c}$ AND $h$ ON THE INTER-EVENT TIMES AND THE DECAY RATE (LOWER BOUND AND NUMERICAL ESTIMATION OF THE DECAY RATE ARE IN BRACKETS).

$p_{1}$ and $p_{2}$, Assumptions 1 and 2 are satisfied with functions $\underline{\alpha}(s)=\min \left(p_{1}, p_{2}\right) s, \bar{\alpha}(s)=\max \left(p_{1}, p_{2}\right) s, \alpha(s)=\lambda_{1} s$, $\lambda_{1}:=\min \left(2 c, 2 a\left(1-\frac{2 p_{1}}{5 p_{2}}\right)-18,2-\frac{4 p_{1} a}{5 p_{2}}-\frac{25 a}{6 p_{1} p_{2}}\right)$, $\beta_{e}^{2}(e)=\frac{2 p_{1}}{5 a}|e|^{2}, H(x)=\sqrt{\frac{2 p_{1} a}{5}}\left(\left|x_{1}\right|+\left|x_{2}\right|\right), \delta\left(y_{p}\right)=18 y_{p}^{2}$, $\theta^{2}=0.6\left(p_{1} a+p_{2} b\right)^{2}, L_{1}=0, L_{2}=1$. Due to the static controller, it is sufficient to consider the error signal $e(t)=y_{p}\left(t_{k}\right)-y_{p}(t)$. The function $\sigma$ of the sampling algorithm (13) is given by $\sigma(\zeta)=\sigma_{c} \zeta^{2}$ with $0<\sigma_{c}<\lambda_{1} \min \left(p_{1}, p_{2}\right)$, and we select $\beta_{V}(s)=\frac{\sigma_{c}}{\min \left(p_{1}, p_{2}\right)} s$. Therefore, the global exponential stability of the eventtriggered implementation of the control system follows from Theorem 2. The decay rate $\gamma$ is obtained by solving the equation $2 \gamma=\lambda_{1}-\frac{\sigma_{c}}{\min \left(p_{1}, p_{2}\right)} e^{2 \gamma h}$ for a given $h>0$. Let consider the parameter values $a=10, b=28, c=8 / 3$ used in [24], then we set $p_{1}=3, p_{2}=3 a, \eta=0.03, \lambda=0.04$ and it is obtained $\mathcal{T}(\eta, \theta, \lambda, L)=0.0021$. Table I provides the average of all the inter-event times of 100 executions of the system with random initial conditions ${ }^{3}\|x(0)\| \leq 10$, a simulation time of $10 \mathrm{~s}, T=0.002$, and several values of the design parameters. In addition, the lower bound of the decay rate is also shown in Table I. It can be observed that an increment on both $\sigma_{c}$ and $h$ leads to an increment of the inter-event times at the expense of reducing the speed of convergence. It should be pointed out that the event-triggering condition proposed in [1] can be directly recovered by setting $\sigma_{c}=0$. In this case, the decay rate is lower bounded by $\lambda_{1}=0.73$ and the average of all the inter-event times of the 100 executions of the system is 0.0078 . As Table I illustrates, the main advantage of the proposed algorithm is that it allows to obtain greater inter-event times in average at the expense of reducing the decay rate.

\section{B. LTI example with stable plant}

In this example, we consider a control system studied in [3], where the matrices are given by

$$
\begin{gathered}
A_{p}=\left[\begin{array}{cc}
0 & 1 \\
-2 & -3
\end{array}\right], B_{p}=B_{w}=\left[\begin{array}{l}
0 \\
1
\end{array}\right], C_{p}=\left[\begin{array}{ll}
1 & 0
\end{array}\right], \\
A_{c}=\left[\begin{array}{cc}
-2 & 1 \\
-13 & -2
\end{array}\right], \quad B_{c}=\left[\begin{array}{l}
-2 \\
-5
\end{array}\right], \quad C_{c}=\left[\begin{array}{ll}
5 & 2
\end{array}\right] .
\end{gathered}
$$

The system is affected by a disturbance $w$ and in order to measure its impact on the system, the performance variable $z$ is defined by the matrix $C_{z}=\left[\begin{array}{llll}1 & 0 & 0 & 0\end{array}\right]$.

\footnotetext{
${ }^{3}$ The initial conditions are taking inside a ball for the sake of the reproducibility of the results.
} 


\begin{tabular}{lcccc}
\hline$\|w\|_{\infty}$ & 0.5 & 1 & 5 & 10 \\
\hline [3], $\tau_{\text {avg }}$ of $y_{p}$ & 0.488 & 0.200 & 0.042 & 0.025 \\
[3], $\tau_{\text {avg }}$ of $y_{c}$ & 0.169 & 0.093 & 0.025 & 0.017 \\
[3], $N_{\tau}$ & 237 & 429 & 1898 & 2884 \\
Proposed, $\tau_{\text {avg }}$ & \multicolumn{4}{c}{0.12} \\
Proposed, $N_{\tau}$ & \multicolumn{4}{c}{496} \\
\hline
\end{tabular}

TABLE II

SIMULATION COMPARISON WITH [3].

Let set the matrix $U_{s}=\left[\begin{array}{cc}1-u & 0 \\ 0 & u\end{array}\right]$ with $u \in(0,1)$, then it can be expected that for a given $\sigma_{c}$ higher inter-event times can be obtained by minimizing $\left\|U_{e}\right\|$. Hence, let set the following minimization problem with decision variables $P, U_{e}$ and $U_{w}$

$$
\left\{\begin{array}{l}
\min \alpha\left\|U_{w}\right\|+(1-\alpha)\left\|U_{e}\right\| \\
\text { subject to }(30),(31), \text { and }(32)
\end{array}\right.
$$

where $\varsigma_{1}=1$ and $\varsigma_{2}=1 \cdot 10^{-5}$ in (31) and (32). The parameter $\alpha$ allows to indirectly balance between the interevent times and the $\mathcal{L}_{\infty}$-gain. Considering the value $\alpha=0.9$, $\gamma=0.402, \sigma_{c}=0.132, h=1$, and $u=0.01$, the optimization problem is solved with $U_{e}=1.56 I, U_{w}=0.21$. Using the results from the optimization problem, and taking $\eta=10$ and $\lambda=0.11$, Proposition 1 applies. Therefore, the event-triggered control system is globally exponentially stable and the $\mathcal{L}_{\infty^{-}}$ gain is smaller or equal to 0.46 . The minimum inter-event time is given by $\mathcal{T}(\eta, 1, \lambda, L)=5.19 \cdot 10^{-6}$. For comparison purpose, we consider the results in [3], which provides the same upper bound of the $\mathcal{L}_{\infty}$-gain. In order to compare both triggering mechanism, let consider the disturbance $w(t)=$ $\|w\|_{\infty} \sin \left(\frac{\pi}{2} t\right)$, zero initial condition and a simulation time of 30s. Table II provides the obtained average inter-event times and the number of triggering events, respectively denoted as $\tau_{\text {avg }}$ and $N_{\tau}$, for several values of $\|w\|_{\infty}$. The number of the triggering events is considered as the sum of the sampling of $y_{p}$ and $y_{c}$. We notice that the number of triggering events significantly increases with the increment of $\|w\|_{\infty}$ for the sampling mechanism in [3], while it remains constant for the proposed mechanism.

\section{LTI example with unstable plant}

Let now consider a control system (Example 2 in [3]) composed by a plant and a controller with matrices

$$
\begin{aligned}
& A_{p}=\left[\begin{array}{cc}
0 & 1 \\
-2 & 3
\end{array}\right], B_{p}=\left[\begin{array}{l}
0 \\
1
\end{array}\right], C_{p}=\left[\begin{array}{ll}
-1 & 4
\end{array}\right], \\
& A_{c}=\left[\begin{array}{cc}
0 & 1 \\
0 & -5
\end{array}\right], B_{c}=\left[\begin{array}{l}
0 \\
1
\end{array}\right], C_{c}=\left[\begin{array}{cc}
1 & -4
\end{array}\right] .
\end{aligned}
$$

Let set the matrix $U_{s}=\left[\begin{array}{cc}0.01 & 0 \\ 0 & 0.99\end{array}\right]$. As aforementioned, we can expect that the maximization of $\sigma_{c}$ and minimization of $\left\|U_{e}\right\|$ lead to greater inter-event times, and thus, it is of interest to maximize $J:=\frac{\sigma_{c}}{\left\|U_{e}\right\|}$. Table III provides the maximum $J$ and $\mathcal{T}(10,1,0.024, L)$ as a function of $N$ obtained by Proposition 2 with $\Upsilon=\left[\begin{array}{llll}1 & 0 & 0 & 0 \\ 0 & 1 & 0 & 0\end{array}\right]$. In addition, it is shown the average of the inter-event times from 100 executions of the system with random initial conditions and a simulation time

\begin{tabular}{lcccc}
\hline $\mathrm{N}$ & 2 & 10 & 20 & 40 \\
\hline $\max J$ & $7 \cdot 10^{-4}$ & $35 \cdot 10^{-4}$ & $40 \cdot 10^{-4}$ & $41 \cdot 10^{-4}$ \\
$\mathcal{T}$ & $1.1 \cdot 10^{-7}$ & $6.33 \cdot 10^{-7}$ & $6.58 \cdot 10^{-7}$ & $6.66 \cdot 10^{-7}$ \\
$\tau_{\text {avg }}$ & 0.0269 & 0.0472 & 0.0489 & 0.0494 \\
\hline
\end{tabular}

TABLE III

EFFECT OF THE NUMBER OF REGIONS $N$ ON THE INTER-EVENT TIMES.

of 40s, where the parameters of the proposed algorithm $h=2$, $U_{e}$ is set to provide the maximum $J$ and $\sigma_{c}$ is set with the greatest value that provides a decay rate $\gamma=0.005$. It can be seen that greater values of $J$ are obtained by increasing the number of regions, which indirectly entails an improvement of the inter-event times.

\section{CONCLUSIONS}

This work proposed an event-triggering mechanism that guarantees the asymptotic/exponential stability and the inputto-output stability of event-triggered control systems, where both the plant output and control output are subject to sampling. The proposed sampling criterion mixes a condition based on the history of the outputs and a dwell-time constraint. Both nonlinear and linear systems are analyzed. For LTI systems the conditions for exponential stability are given in the form of LMI, and in addition, we provided a procedure to obtain un upper bound of the $\mathcal{L}_{\infty}$-gain. Several numerical examples showed how the inter-event times can be increased by a suitable design of the parameters, but at the price of reducing the convergence rate of the trajectories. For the future work, it could be interesting to consider asynchronous sampling and to apply the proposed mechanism to multi-agent systems.

\section{APPENDIX}

Lemma 1: (Halanay's Inequality [7]) Let $\psi:[-h, \infty) \rightarrow$ $\mathbb{R}_{+}$be bounded on $[-h, 0], h>0$ and continuous on $[0, \infty)$. Assume that for some positive constants $\lambda_{2}<\lambda_{1}$ the following inequality holds:

$$
\dot{\psi} \leq-\lambda_{1} \psi(t)+\lambda_{2} \max _{s \in[t-h, t]} \psi(s), t \geq 0
$$

then $\psi(t) \leq e^{-\gamma t} \max _{s \in[-h, 0]} \psi(s), t \geq 0$, where $\gamma>0$ is the unique positive solution of the equation $\gamma=\lambda_{1}-\lambda_{2} e^{\gamma h}$.

Proposition 3: Consider a continuous and differentiable almost everywhere function $\psi:[-h, \infty) \rightarrow \mathbb{R}_{+}$satisfying

$$
\dot{\psi}(t) \leq-v_{1}(\psi(t))+v_{2}\left(\left\|\psi_{t}\right\|\right),
$$

whenever $\psi(t) \geq \mu$ and $t \geq 0$, for some $\mu>0$ and functions $v_{1}$ and $v_{2}$. In addition, assume there exists a continuous, nondecreasing function $\rho(s)>s$ such that $v(s):=v_{1}(s)-$ $v_{2}(\rho(s))$ is nondecreasing and $v(s)>0$ for all $s>0$, then

1. $\psi(t) \leq \max \left(\mu, \psi(0), \rho^{-1}\left(\left\|\psi_{0}\right\|\right)\right), t \geq 0$,

2. there exists $T=T(\vartheta, \mu)$ such that if $\left\|\psi_{0}\right\| \leq \vartheta$ then $\psi(t) \leq \mu$ for all $t \geq T$ and for all $\vartheta>0$.

Proof: First we prove the Statement 1. To do so, let consider the following three cases for some $\check{t} \geq 0$ : 
Case 1: suppose that $\psi(\check{t})<\mu$ then there exists $\hat{t}>\check{t}$ such that $\psi(t) \leq \mu$ for all $t \in[\check{t}, \hat{t}]$.

Case 2: suppose that $\psi(\check{t})<\rho^{-1}\left(\left\|\psi_{\breve{t}}\right\|\right)$ then there exists $\hat{t}>\check{t}$ such that $\psi(t)<\rho^{-1}\left(\left\|\psi_{\breve{t}}\right\|\right)$ for all $t \in[\check{t}, \hat{t}]$.

Case 3: suppose that $\rho^{-1}\left(\left\|\psi_{\breve{t}}\right\|\right) \leq \psi(\check{t})$ and $\mu \leq \psi(\check{t})$ then (47) leads to $\dot{\psi}(\check{t}) \leq-v(\psi(\check{t})) \leq 0$. Hence, it is impossible for $\psi(t)$ to exceed $\psi(\check{t})$, impliying $\psi(t) \leq \psi(\check{t})$ for all $t \geq \check{t}$.

The rest of the proof follows from the combination of the three cases for all $t \geq 0$.

Now let focus on the Statement 2. For given $\vartheta, \mu>0$, suppose that $\mu<\vartheta$, otherwise Statement 1 implies Statement 2 with $T(\vartheta, \mu)=0$. The continuity of $\rho$ implies that there exists an $a>0$, such that $a<s-\rho^{-1}(s)$ for $\mu \leq s \leq \vartheta$. In addition, let $\nu:=\min _{\mu \leq s \leq \vartheta} v(s)$. Now consider the time $\tau_{0} \in[-h, 0]$ such that $\psi\left(\tau_{0}\right)=\left\|\psi_{0}\right\|$, then there are two cases:

Case 1: If $\rho^{-1}\left(\psi\left(\tau_{0}\right)\right)>\psi(0)$, then Statement 1 implies $\psi(t) \leq \max \left(\rho^{-1}\left(\psi\left(\tau_{0}\right)\right), \mu\right) \leq \max \left(\psi\left(\tau_{0}\right)-a, \mu\right), \forall t \geq 0$.

Case 2: suppose that $\rho^{-1}\left(\psi\left(\tau_{0}\right)\right) \leq \psi(0)$. If $\psi(0)>\mu$, then there exists a scalar $d$ with $0<d \leq \frac{a}{\nu}$ such that $\psi(t) \leq$ $\rho(\psi(t))$ for all $\psi \in[0, d]$, and thus (47) leads to $\dot{\psi}(t) \leq$ $-v(\psi(t)) \leq-\nu, t \in[0, d]$. From Statement 1, it follows $\psi(t) \leq \max \left(\psi\left(\tau_{0}\right)-a, \mu\right), \forall t \geq d$. Hence, both cases lead to $\psi(t) \leq \max \left(\psi\left(\tau_{0}\right)-a, \mu\right), \forall t \geq \frac{a}{\nu}$. If $\psi\left(\tau_{0}\right)-a \leq \mu$ then Statement 2 is proved, otherwise let pick a time $\tau_{1} \in$ $\left[\tau_{0}+\frac{a}{\nu}, \tau_{0}+\frac{a}{\nu}+h\right]$ such that $\psi\left(\tau_{1}\right)=\left\|\psi_{\tau_{0}+\frac{a}{\nu}+h}\right\|$. Note that $\tau_{1} \geq \tau_{0}+\frac{a}{\nu}$. Following the reasoning of $\tau_{0}$ we obtain $\psi(t) \leq \max \left(\psi\left(\tau_{1}\right)-a, \mu\right), \forall t \geq \tau_{0}+\frac{a}{\nu}+h$. The process can be repeated for a sequence of times $\tau_{0}<\tau_{1}<\tau_{2}<\cdots<\tau_{k}$ as long as $\psi\left(\tau_{k}\right) \geq \mu$. Therefore, for a large enough $k$, there exists time $T \geq \tau_{k}$ such that $\psi(T) \leq \mu$ and Statement 1 implies $\psi(t) \leq \mu$ for all $t \geq T$.

Lemma 2: For each pair of Lipschitz continuous functions $v_{1}$ and $v_{2}$ such that there exists a continuous, nondecreasing function $\rho(s)>s$ satisfying that $v(s):=v_{1}(s)-v_{2}(\rho(s))$ is a nondecreasing function and $v(s)>0$ for all $s>0$, there exists a function $\beta \in \mathcal{K} \mathcal{L}$ with the following property: if $\psi:[-h, \infty) \rightarrow \mathbb{R}_{+}$is a continuous and differentiable almost everywhere function that satisfies (47) for some given $\mu>0$, then it holds that $\psi(t) \leq \max \left(\beta\left(\left\|\psi_{0}\right\|, t\right), \mu\right)$ for all $t \geq 0$.

Proof: The proof is divided in five steps:

1) Existence of a global solution to $\dot{y}(t)=-v_{1}(y(t))+$ $v_{2}\left(\left\|y_{t}\right\|\right)$. The proof follows from the Lipschitz continuity of $v_{1}$ and $v_{2}$ and the results in [6].

2) Existence of $T(\vartheta, \mu)$ such that if $\left\|y_{0}\right\| \leq \vartheta$ then $y(t) \leq$ $\mu, \forall t \geq T(\vartheta, \mu)$. It follows by Proposition 3 .

3) Comparison principle: $y_{0}=\psi_{0}$ implies $\psi(t) \leq y(t)$, $\forall t \geq 0$. In order to prove it, let define the continuous function $d(t):=\psi(t)-y(t)$. Assume $y_{0}=\psi_{0}$ then $d(t)=0, \forall t \in[-h, 0]$. By way of contradiction, suppose that $d(t)>0$ for all $t \in(0, \varepsilon)$ for some $\varepsilon>0$. Considering $\varepsilon$ small enough, it follows $\dot{d}(t)>0$ for all $t \in(0, \varepsilon)$. Let now define $\bar{t}:=\sup \{t \in[-h, 0]$ : $\left.y(t)=y_{0}\right\}$. If $\bar{t}<0$ then $\left\|y_{t}\right\|=\left\|\psi_{t}\right\|$ for all $t \in[0, \varepsilon)$. On the contrary, if $\bar{t}=0$ then Proposition 3 implies $\dot{y}(t)<0$ and $\dot{\psi}(t)<0$ for all $t \in[0, \varepsilon)$, and thus, $\left\|y_{t}\right\|=\left\|\psi_{t}\right\|=\left\|y_{0}\right\|$ for all $t \in[0, \varepsilon)$. Therefore, from (47), it is obtain $\dot{d}(t) \leq-v_{1}(\psi(t))+v_{1}(y(t))<0$, which is a contradiction. Therefore, $d(t) \leq 0$ for all $t \in[0, \varepsilon)$. Repeating the same procedure, it can be proved that $d(t) \leq 0$ for all $t \in[0, \infty)$.

4) Steps 2 and 3 lead to $\psi(t) \leq y(t) \leq \mu$ for all $t \geq$ $T(\vartheta, \mu)$ whenever $\left\|\psi_{0}\right\|=\left\|y_{0}\right\| \leq \vartheta$.

5) Construction of the function $\beta \in \mathcal{K} \mathcal{L}$ from $T(\vartheta, \mu)$. The proof follows as the proof in [11] (Appendix C.6).

\section{REFERENCES}

[1] M. Abdelrahim, R. Postoyan, J. Daafouz, and D. Nešić. Input-to-state stabilization of nonlinear systems using event-triggered output feedback controllers. In 14th European Control Conf., pages 2185-2190, Linz, Austria, 2015.

[2] M. A. Davó, M. Fiacchini, and C. Prieur. Output memory-based eventtriggered control. In IEEE Conf. on Dec. and Cont., Las Vegas, USA, 2016.

[3] M.C.F. Donkers and W.P.M.H. Heemels. Output-based event-triggered control with guaranteed $L_{\infty}$-gain and improved and decentralised eventtriggering. IEEE Trans. Automat. Control, 57(6):1362-1376, 2012.

[4] F. Forni, S. Galeani, D. Nešić, and L. Zaccarian. Event-triggered transmission for linear control over communication channels. Automatica, 50(2):490-498, 2014.

[5] E. Fridman. Introduction to Time-Delay Systems. Systems \& Control: Foundations \& Applications. Birkhäuser, 2014.

[6] L. Georgiev and V. Angelov. On the existence and uniqueness of solutions for maximum equations. Glasnik Matematicki, 37(2):275-281, 2002.

[7] A. Halanay. Differential equation: stability, oscillations, time lags. Academic, New York, 1966.

[8] W.P.M.H. Heemels, K.H. Johansson, and P. Tabuada. An introduction to event-triggered and self-triggered control. In Proc. 51st IEEE Conference on Decision and Control, pages 3270-3285, Maui, HI, 2012.

[9] E. Hendricks, M. Jensen, A. Chevalier, and T. Vesterholm. Problems in event based engine control. In American Control Conf., pages 1585$1587,1994$.

[10] M. Johansson and A. Rantzer. Computation of piecewise quadratic Lyapunov functions for hybrid systems. IEEE Trans. Automat. Control, 43(4):555-559, 1998.

[11] H.K. Khalil. Nonlinear Systems. Prentice-Hall, 3rd edition, 2002.

[12] D. Lehmann and J. Lunze. Event-based output-feedback control. In 19th Med. Conf. on Control and Automat., pages 982-987, June 2011.

[13] D. Lehmann and J. Lunze. Extension and experimental evaluation of an event-based state-feedback approach. Control Engineering Practice, 19:101-112, 2011.

[14] J. Lunze and D. Lehmann. A state-feedback approach to event-based control. Automatica, 46(1):211-215, 2010.

[15] M. Miskowicz. Event-based control and signal processing. CRC Press/Taylor \& Francis Group, 2015.

[16] D. Nešić, A. R. Teel, and D. Carnevale. Explicit computation of the sampling period in emulation of controllers for nonlinear sampled-data systems. IEEE Transactions on Automatic control, 54(3):619-624, 2009.

[17] C. Peng and Q.L. Han. Output-based event-triggered $H_{\infty}$ control for sampled-data control systems with nonuniform sampling. In American Control Conf., pages 1727-1732, Washington, June 2013.

[18] R. Postoyan, P. Tabuada, D. Nesic, and A. Anta. A framework for the event-triggered stabilization of nonlinear systems. IEEE Transactions on Automatic Control, 60(4):982-996, 2015.

[19] J. H. Sandee, W. P. M. H. Heemels, S. B. F. Hulsenboom, and P. P. J. van den Bosch. Analysis and experimental validation of a sensor-based event-driven controller. In American Control Conf., pages 2867-2874, New York City, USA, 2007.

[20] A. Seuret, C. Prieur, and N. Marchand. Stability of non-linear systems by means of event-triggered sampling algorithms. IMA Journal of Mathematical Control and Information, 31(3):415-433, 2014.

[21] P. Tabuada. Event-triggered real-time scheduling of stabilizing control tasks. IEEE Transactions on Automatic Control, 52(9):1680-1685, 2007.

[22] A. Tanwani, C. Prieur, and M. Fiacchini. Observer-based feedback stabilization of linear systems with event-triggered sampling and dynamic quantization. Systems \& Control Letters, 94:46-56, 2016.

[23] A. Tanwani, A.R. Teel, and C. Prieur. On using norm estimators for event-triggered control with dynamic output feedback. In IEEE Conf. on Dec. and Control, pages 5500-5505, Osaka, Japan, 2015.

[24] C. Wan and D. Bernstein. Nonlinear feedback control with global stabilization. Dynamics and Control, 5:321-346, 1995. 